\title{
Slow Water Diffusion in Micellar Solutions
}

\author{
Szabolcs Vass, ${ }^{*},{ }^{\dagger}$ Hans Grimm, ${ }^{\ddagger}$ István Bányai, ${ }^{\S}$ Gerhard Meier, ${ }^{\ddagger}$ and Tíbor Gilányi" \\ KFKI Atomic Energy Research Institute, P.O. Box 49, H-1525 Budapest, Hungary, Institute for Solid State \\ Physics, Jülich Research Center, D-52425 Jülich, Germany, Department of Physical Chemistry, University of \\ Debrecen, P.O. Box 7, H-4010 Debrecen, Hungary, and Department of Colloid Chemistry, Loránd Eötvös \\ University, P.O. Box 32, H-1518 Budapest, Hungary
}

Received: April 4, 2005

\begin{abstract}
Slowly diffusing water molecules were found by quasi-elastic neutron scattering (QENS) in a sodium dodecyl sulfate (SDS) micellar solution, and both their diffusion coefficient $\left(4.33 \times 10^{-6} \mathrm{~cm}^{2} \cdot \mathrm{s}^{-1}\right)$ and mole fraction (0.057) were determined. After successfully checking the mean slowing down of solvent molecules by the gradient compensated stimulated spin-echo (GCSTE) pulse sequence NMR method, a similar effect was observed with this technique in the solvent phase of dodecyl trimethylammonium bromide (DTAB) and differing chain length $(X=12,20,30$, and 40) ethoxylated nonyl phenol (9NX) micellar systems. Following the literature, the experimental results are qualitatively explained by assuming that, apart from ionic hydration, $\mathrm{H}$-bonds may form between the solvent molecules and the $\mathrm{O}$ or $\mathrm{N}$ atoms present in the hydrophilic (head)groups of the micelle-forming monomers.
\end{abstract}

\section{Introduction}

Molecular dynamics in water and aqueous solutions is subject to extensive experimental studies carried out by classical (viscosity and diffusion measurements) as well as modern techniques (NMR, dielectric relaxation, and dynamic light and neutron scattering). In bulk water, quasi-elastic neutron scattering (QENS) resulted in two relaxation times, both in the $10^{-12}$ $\mathrm{s}$ range at room temperature. ${ }^{1,2}$ One of them, $\tau_{0}$, stands for the residence time of water molecules in a hypothetical bound state ${ }^{3,4}$ between two free translational jumps; the other, $\tau_{1}$, is characteristic of the rotation ${ }^{5}$ of water molecules.

Solutes locally perturb the structure of aqueous solvents. Analysis of excess thermodynamic quantities led to the assumption that apolar ("hydrophobic") solutes may cause the neighboring water molecules to be more "structured" in comparison with the bulk, ${ }^{6}$ and this change may affect the degrees of freedom of their motion. With the different types of biomolecules present in the system, dielectric ${ }^{7,8}$ and NMR relaxation ${ }^{7,9}$ experiments indicated that $\tau_{1}$ splits into two components. One of them is ultrafast, $\sim 10^{-12}-10^{-11} \mathrm{~s}$, resembling that in the bulk aqueous phase; the other is significantly longer, $\sim 10^{-10}$ $10^{-9} \mathrm{~s}$. This splitting is thought to arise from strong H-bonds formed between the hydrated ("biological") water and certain segments of the slow dynamics biomolecules, thereby hindering the rotation of the water molecules involved. ${ }^{7,8}$

Molecular confinements, which cause slowing down of about 2 orders of magnitude in the rotational relaxation of water molecules, are expected not to leave their translation unaffected.

\footnotetext{
* Corresponding author. E-mail: szvass@ sunserv.kfki.hu.

KFKI Atomic Energy Research Institute.

Jülich Research Center.

$\S$ University of Debrecen.

" Loránd Eötvös University.
}

Significantly hindered diffusivity of water molecules has been observed in (dense) solutions of small organic molecules with polar and apolar segments ${ }^{10-12}$ by QENS and in various mesoscopic systems (micellar solutions ${ }^{13-15}$ ) by gradient compensated stimulated spin-echo (GCSTE) NMR. Although GCSTE NMR indicates that the mean diffusion coefficient of water molecules decreases in the presence of micelles, this method is unable to distinguish between water molecules of different diffusivities. In the present work, an attempt is made to determine by QENS the mole fraction and the diffusion coefficient of slow (and bulk) water molecules in the solvent phase of an SDS micellar solution. GCSTE NMR measurements were made in sodium dodecyl sulfate (SDS), dodecyl trimethylammonium bromide (DTAB), and ethoxylated nonyl phenol (9NX) micellar solutions partly to confirm the QENS results obtained in the SDS solution and partly to compare the hydration in anionic, cationic, and nonionic micellar systems.

\section{Experimental Section}

2.1. QENS in SDS Micellar Solution. QENS experiments were carried out on the high-resolution (energy, 1.2-2.4 $\mu \mathrm{eV}$ in the energy transfer range $[-17,17] \mu \mathrm{eV}$; momentum transfer, $\sim 0.5-0.8 \mathrm{~nm}^{-1}$ for $Q$ 's of $1.6,2.4$, and $3.2 \mathrm{~nm}^{-1}$ ) backscattering spectrometer at the FRJ2 reactor of the Jülich Research Center, Germany. $\mathrm{Si}(111)$ crystals served as the monochromator (moving) and the analyzer (at rest) corresponding to the final wavelength $\lambda=0.62718 \mathrm{~nm}$. The liquid sample was poured between two thin-walled $(0.5 \mathrm{~mm})$ aluminum cylinders, and the sample thickness was $\sim 0.2 \mathrm{~mm}$. Each sample was measured both in liquid (at $309 \pm 3 \mathrm{~K}$ ) and in the frozen state (at $\sim 260$ $\mathrm{K}$ ), and the latter served as a reference for the background determination. Spectra were collected for $48 \mathrm{~h}$ (liquid) or $24 \mathrm{~h}$ (frozen samples). The energy resolution of the spectrometer was determined by a vanadium spectrum.

(C) 2005 American Chemical Society 
TABLE 1: Values of the Parameter Combination $X_{\mathrm{w}} C_{\mathrm{w}}(Q)$ and $X_{\mathrm{m}} C_{\mathrm{m}}(Q)$ for $\mathrm{H}_{2} \mathrm{O}\left(X_{\mathrm{w}}=1, X_{\mathrm{m}}=0\right)$ and for $0.2 \mathrm{M}$ Solutions $\left(X_{\mathrm{w}} \approx 1, X_{\mathrm{m}}=5.15 \times 10^{-5}\right)$ of $p$-SDS and $d$-SDS in $\mathrm{H}_{2} \mathrm{O}$ in the Three Detector Positions

\begin{tabular}{llccc}
\hline & & \multicolumn{3}{c}{$X_{j} C_{j}(Q)\left(10^{-24} \mathrm{~cm}^{2}\right)$} \\
\cline { 3 - 5 } system & $j$ & $Q=1.61 \mathrm{~nm}^{2}$ & $Q=2.41 \mathrm{~nm}^{2}$ & $Q=3.19 \mathrm{~nm}^{2}$ \\
\hline $\mathrm{H}_{2} \mathrm{O}$ & $\mathrm{w}$ & 12.72 & 12.72 & 12.72 \\
& $\mathrm{~m}$ & & & \\
$d$-SDS & $\mathrm{w}$ & 10.40 & 12.75 & 12.66 \\
& $\mathrm{~m}$ & 16.62 & 0.02 & 0.36 \\
$p$-SDS & $\mathrm{w}$ & 12.75 & 12.75 & 12.75 \\
& $\mathrm{~m}$ & 0.57 & 0.61 & 0.58
\end{tabular}

The scattering cross section $\mathrm{d}^{2} \sigma / \mathrm{d} \Omega \mathrm{d} E$ for an $n$-component liquid was derived from van Hove's formula, ${ }^{16,17}$ by using the convolution approximation to determine its distinct part. ${ }^{18,17}$ The scattering intensity $I(E, Q)$ represents a convolution of the scattering cross section with the instrumental resolution function $R(E)$, and the result is expressed in terms of Lorentzians $L\left(E, Q ; D_{j}\right)=D_{j} \hbar Q^{2} / \pi\left[E^{2}+\left(D_{j} \hbar Q^{2}\right)^{2}\right]$ standing for component $j$ with diffusion coefficient $D_{j}$ :

$$
I(E, Q)=\sum_{j}^{n} A_{j}(Q) L\left(E, Q ; D_{j}\right) * R(E)
$$

where $A_{j}(Q)=X_{j} a(Q) W_{j}(Q) C_{j}(Q)$ is the area of the Lorentzian $\left(X_{j}\right.$ stands for the mole fraction of the component, and $a(Q)$ is the counting efficiency, assumedly equal for all samples). The quantity $W_{j}(Q)=\exp \left(-\left\langle u^{2}\right\rangle Q^{2} / 3\right)$ is the Debye-Waller factor defined by the mean squared vibration amplitude $\left\langle u^{2}{ }_{j}\right\rangle$. In the applied range of $Q$ and $E$, the molecular rotations cause only a small, negligible increase in the width of the Lorentzians. ${ }^{2}$ Finally, $C_{j}(Q)$ is the static differential cross section:

$$
C_{j}(Q)=b_{\mathrm{i}, j}^{2}+\sum_{k}^{n} b_{\mathrm{c}, j} b_{\mathrm{c}, k} S_{j k}(Q)
$$

Here, $b_{\mathrm{c}, j}$ and $b_{\mathrm{i}, j}^{2}$ respectively stand for the coherent and squared incoherent scattering length of component $j$ and $S_{j k}(Q)$ is the static structure factor of components $j$ and $k$; in what follows, the subscript $j=\mathrm{w}$ stands for water and $j=\mathrm{m}$ for micelles.

The experiments were carried out in bulk $\mathrm{H}_{2} \mathrm{O}$ and in $0.2 \mathrm{M}$ $\left(\mathrm{mol} \cdot \mathrm{dm}^{-3}\right) \mathrm{H}_{2} \mathrm{O}$ solutions of protonated $(p)$ and $98 \%$ deuterated (d) $\mathrm{SDS}$ and of $\mathrm{NaHSO}_{4}$ (for the preparation of $p$-SDS, see ref $19 ; d$-SDS was purchased from Cambridge). The $X_{j} C_{j}$ values for water and micelles are listed in Table 1; scattering length data were taken from ref 20. Because $D_{j}$ is independent of $Q$ in the applied range of $E,{ }^{1,2,21}$ the backscattering patterns (corrected for background and empty sample holder) from all three detectors were fitted by eq $1 \mathrm{a}$ in the same session.

2.1.1. Results from Bulk $\mathrm{H}_{2} \mathrm{O}$. Equation 1a was evaluated for a single component. Since ${ }^{20} b_{\mathrm{i}, \mathrm{w}}^{2} \gg b_{\mathrm{c}, \mathrm{w}}^{2}$ and $C_{\mathrm{w}} \approx b_{\mathrm{i}, \mathrm{w}}^{2}$, having ${ }^{2}$ $\left\langle u_{\mathrm{w}}^{2}\right\rangle \sim 0.0025 \mathrm{~nm}^{-2}, a(Q) \approx A_{\mathrm{w}}^{0}(Q) / W_{\mathrm{w}}(Q) b^{2}{ }_{\mathrm{i}, \mathrm{w}}$ was determined for further use. The diffusion coefficient $D^{0}{ }_{\mathrm{w}}$ of $\mathrm{H}_{2} \mathrm{O}$ molecules in bulk water is $2.98 \times 10^{-5} \mathrm{~cm}^{2} \cdot \mathrm{s}^{-1}$ at $309 \mathrm{~K}$, in agreement with the value $(2.94 \pm 0.03) \times 10^{-5} \mathrm{~cm}^{2} \cdot \mathrm{s}^{-1}$, calculated from literature data. ${ }^{22}$

2.1.2. Results from $d-S D S / H_{2} O$ Micellar Solutions. The difficulty in evaluating backscattering patterns from micellar solutions is the calculation of the micelle/micelle and micelle/ water structure factors. The former is obtained from a standard procedure, ${ }^{23}$ and the latter is approximated by assuming that the micelle/water pair-correlation function is a step function. The strong coherent contrast of $d$-SDS micelles in $\mathrm{H}_{2} \mathrm{O}$ causes both $C_{\mathrm{w}}$ and $C_{\mathrm{m}}$ to significantly depend on $Q$ (cf. Table 1 ).
Using the known values of $A^{0}{ }_{\mathrm{w}}, D^{0}{ }_{\mathrm{w}}$, and $C_{\mathrm{w}}$, the contribution from the aqueous phase is fixed in the model function:

$$
\begin{aligned}
& I(E, Q) \approx \\
& {\left[\frac{A^{0}{ }_{\mathrm{w}} X_{\mathrm{w}} C_{\mathrm{w}}(Q)}{b^{2}{ }_{\mathrm{i}, \mathrm{w}}} L\left(E, Q, D_{\mathrm{w}}^{0}\right)+A_{\mathrm{m}}(Q) L\left(E, Q, D_{\mathrm{m}}\right)\right] * R(E)}
\end{aligned}
$$

The fit resulted in $D_{\mathrm{m}}=9.61 \times 10^{-7} \mathrm{~cm}^{2} \cdot \mathrm{s}^{-1}$; the values published in the literature are from neutron spin- $\mathrm{echo}^{24}$ (NSE) $\sim 7 \times 10^{-7}$ and from dynamic light scattering ${ }^{25} \sim 1.4 \times 10^{-6}$ $\mathrm{cm}^{2} \cdot \mathrm{s}^{-1}$.

2.1.3. Determination of Slow Water in $\mathrm{p}-\mathrm{SDS} / \mathrm{H}_{2} \mathrm{O}$ Micellar Solutions. Due to the weak coherent contrast of $p$-SDS micelles in $\mathrm{H}_{2} \mathrm{O}$ and the small $b_{\mathrm{c}, \mathrm{w}}$ value of $\mathrm{H}_{2} \mathrm{O}$, the terms consisting of $S_{\mathrm{wm}}(Q)$ and $S_{\mathrm{mm}}(Q)$ contribute perceptively neither to $C_{\mathrm{w}}(Q)$ nor to $C_{\mathrm{m}}(Q)$ (see Table 1 ); the information on the movement of $p$-SDS micelles stems from the incoherent scattering of $\mathrm{H}$ atoms in the alkyl chain. The contribution from water is divided into the usual bulk and the hypothetical slow components:

$$
\begin{aligned}
& I(E, Q) \approx \frac{A^{0}{ }_{\mathrm{w}}}{b^{2}{ }_{\mathrm{i}, \mathrm{w}}}\left[C _ { \mathrm { w } } ( Q ) \left\{(1-\alpha) L\left(E, Q ; D^{0}{ }_{\mathrm{w}}\right)+\right.\right. \\
& \left.\left.\alpha L\left(E, Q ; D_{\mathrm{w}}^{\mathrm{s}}\right)\right\}+\frac{W_{\mathrm{m}}(Q)}{W_{\mathrm{w}}(Q)} X_{\mathrm{m}} C_{\mathrm{m}}(Q) L\left(E, Q ; D_{\mathrm{m}}\right)\right] * R(E)
\end{aligned}
$$

where the diffusion coefficients of bulk water molecules and micelles were respectively fixed at the best-fit values $D^{0}{ }_{\mathrm{w}}$ and $D_{\mathrm{m}}$; since no data were found for dodecyl chains, $W_{\mathrm{m}}(Q)$ was calculated by assuming $\left\langle u_{\mathrm{m}}^{2}\right\rangle \approx 0.01 \mathrm{~nm}^{2}$, the mean squared displacement of poly(didecyl itaconate) ${ }^{26}$. The free-fitting parameters are $D^{\mathrm{s}}{ }_{\mathrm{w}}$, the diffusion coefficient, and $\alpha=X_{\mathrm{w}}^{\mathrm{s}} / X_{\mathrm{w}}$, the fraction of slow water molecules in the solvent. Backgroundcorrected raw data and the best-fit curves are plotted in Figure 1 ; the best-fit values of the fitting parameters are $D^{\mathrm{s}}{ }_{\mathrm{w}}=4.33$ $\times 10^{-6} \mathrm{~cm}^{2} \cdot \mathrm{s}^{-1}$ and $\alpha=0.057$.

2.1.4. Unsuccessful Attempt to Find Slow Water in $\mathrm{NaHSO}_{4}$ Solution. The $0.2 \mathrm{M} \mathrm{NaHSO}_{4}$ solution serves as a model for studying the contribution to the slowing down of solvent molecules from the hydrate spheres of the counterions and, to a certain extent, that from the headgroup ions. Its backscattering patterns were also fitted by eq 3 , after having fixed the diffusion coefficient of bulk water molecules at $D^{0}{ }_{\mathrm{w}}$ and set $X_{\mathrm{m}}=0$. The fit resulted for the slow diffusion coefficient in a value which differs from the bulk one by less than $1 \%$ such that the best-fit value of $\alpha$ turned out to be ambiguous.

2.2. NMR in Micellar Solutions of SDS, DTAB, and Ethoxylated Nonyl Phenols with 12, 20, 30, and 40 Ethoxy Groups. The QENS results were confirmed by GCSTE NMR. ${ }^{27}$ Among the various branches ${ }^{28,29}$ of the pulsed field gradient spin-echo method, ${ }^{30}$ this special technique is designed for determining the diffusion coefficient $D$ of (small) molecules. The various aspects of the application of NMR in surfactant systems are reviewed in two recent papers; one of them ${ }^{31}$ is more method-oriented, and the other ${ }^{32}$ is more system-oriented. The diffusion coefficient is derived from the change in the integrated intensity $I$ of the component studied

$$
\ln \left(\frac{I}{I_{0}}\right)=-B g^{2}, \quad B=D \gamma^{2} c^{2}\left[\Delta-\frac{\delta}{3}\right]
$$

$I_{0}$ is the integrated intensity without a gradient, $g(=\max 50$ $\mathrm{G} \cdot \mathrm{cm}^{-1}$ ) the magnetic field gradient, $\gamma$ the gyromagnetic constant, $c$ a constant determined by the shape of the gradient 

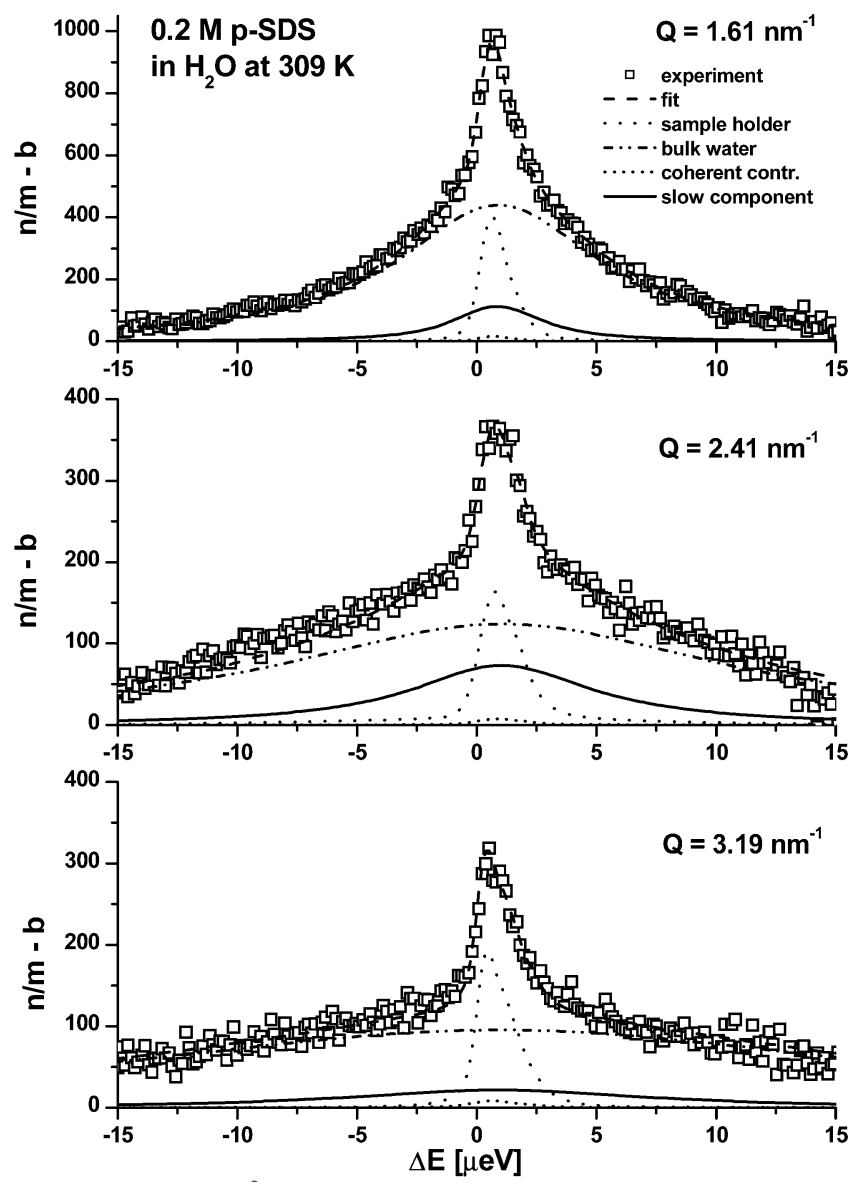

Figure 1. Background-corrected backscattering intensities from a 0.2 $\mathrm{M} \mathrm{H}_{2} \mathrm{O}$ solution of $p$-SDS at $309 \mathrm{~K}$ in detector positions at $Q=1.61$, 2.42 , and $3.19 \mathrm{~nm}^{-1}$

pulse, $\Delta(=50 \mathrm{~ms})$ the time interval between two gradient pulses, and $\delta(=2 \mathrm{~ms})$ the gradient pulse duration. ${ }^{27}$ The measurements were performed at $309 \mathrm{~K}$ on a Bruker DRX 500 spectrometer at the University of Debrecen; the solution concentrations were $0.2 \mathrm{M}$ ( $p$-SDS and DTAB, the latter purchased from Sigma) and $0.04 \mathrm{M}$ (ethoxylated nonyl phenols, purchased from Dow Chemicals). Provided that the exchange between the two types of water is fast on the time scale defined by the chemical shift difference between them, the spectrometer sees $\bar{D}_{\mathrm{w}}$, the average of the two diffusion constants weighted by the molar fractions of the two types of water, ${ }^{29}$ with the notation used in eq 3 :

$$
\bar{D}_{\mathrm{w}}=\alpha D_{\mathrm{w}}^{\mathrm{s}}+(1-\alpha) D_{\mathrm{w}}^{0}
$$

The plots of $\ln \left(I / I_{0}\right)$ for water in bulk, $p$-SDS, and $9 \mathrm{~N} 40$ solutions are shown in Figure 2. To confirm the QENS results on the solvent diffusion coefficient by NMR, two methodrelated, systematic errors have to be corrected. The first stems from the different isotopic composition of the solvents. In QENS, the solvent is pure $\mathrm{H}_{2} \mathrm{O}$; in NMR, the solvent consists of $5 \mathrm{~mol} \% \mathrm{D}_{2} \mathrm{O}$, causing the effective self-diffusion coefficient of the solvent molecules to decrease to $D^{0}{ }_{\mathrm{w}}=2.91 \times 10^{-5}$ $\mathrm{cm}^{2} \cdot \mathrm{s}^{-1}$ in the bulk. To avoid or, at least, essentially reduce this type of systematic error, the quantity

$$
S_{\text {obsd }}=1-\bar{D}_{\mathrm{w}} / D^{0}{ }_{\mathrm{w}}=\alpha\left(1-D_{\mathrm{w}}^{\mathrm{s}} / D_{\mathrm{w}}^{0}\right)
$$

that we call "observed slowing down" is introduced for comparing NMR and QENS data. Numerical results of $S_{\text {obsd }}$ from NMR are presented in Table 2.

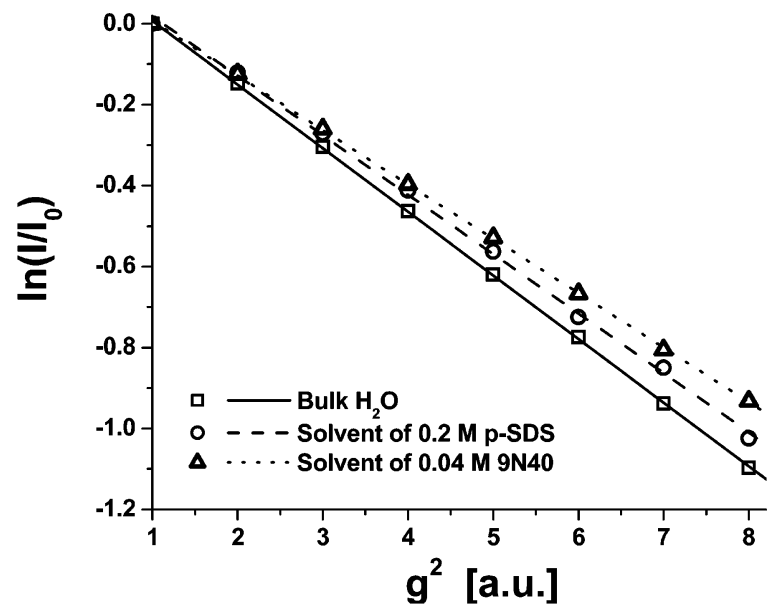

Figure 2. Logarithm of $I / I_{0}$ as a function of $g^{2}$, the squared gradient field strength, for water molecules in the bulk and in the solvent phase of $0.2 \mathrm{M}$ SDS and $0.04 \mathrm{M} 9 \mathrm{~N} 40$ solutions.

TABLE 2: Slowing Down $S_{\text {obsd }}=1-\bar{D}_{\mathrm{w}} / D^{0}{ }_{\mathrm{w}}$ of $\mathrm{H}_{2} \mathrm{O}$ Molecules Observed by GCSTE NMR in Various Types of Micellar Solutions of Volume Fraction $\Phi$ and the Diffusion Coefficient $D_{\mathrm{m}}$ of Micelles Determined by Different Experimental Techniques at $309 \mathrm{~K}$

\begin{tabular}{lcllrll}
\hline & & & \multicolumn{3}{c}{$D_{\mathrm{m}}\left(10^{-7} \mathrm{~cm}^{2} \cdot \mathrm{s}^{-1}\right)$} \\
\cline { 4 - 7 } \multicolumn{1}{c}{ system } & $S_{\text {obsd }}$ & \multicolumn{1}{c}{$\Phi / 2$} & QENS & $\mathrm{NMR}^{a}$ & DLS & NSE \\
\hline$p$-SDS/ $\mathrm{H}_{2} \mathrm{O}$ & $0.062 \pm 0.014$ & $0.024^{34}$ & 9.61 & 8.79 & $14.1^{21}$ & $7.0^{20}$ \\
$\mathrm{DTAB} / \mathrm{H}_{2} \mathrm{O}$ & $0.076 \pm 0.024$ & $0.029^{b}$ & & 10.67 & & \\
$9 \mathrm{~N} 12 / \mathrm{H}_{2} \mathrm{O}$ & $0.034 \pm 0.024$ & $0.013^{c}$ & & 7.68 & & \\
$9 \mathrm{~N} 20 / \mathrm{H}_{2} \mathrm{O}$ & $0.086 \pm 0.024$ & $0.019^{c}$ & & 6.08 & $8.55^{d}$ & \\
$9 \mathrm{~N} 30 / \mathrm{H}_{2} \mathrm{O}$ & $0.103 \pm 0.024$ & $0.027^{c}$ & & 4.71 & $7.68^{d}$ & \\
$9 \mathrm{~N} 40 / \mathrm{H}_{2} \mathrm{O}$ & $0.141 \pm 0.024$ & $0.034^{c}$ & & 2.46 & $7.25^{d}$ &
\end{tabular}

${ }^{a}$ Without correcting for obstruction effects. ${ }^{b}$ Estimated from data published in refs 34 and $35 .{ }^{c}$ Calculated from unpublished apparent molar volumes. ${ }^{d}$ Data measured in $10 \mathrm{~g} \cdot \mathrm{dm}^{-3}\left(9 \mathrm{~N} 20,9.1 \times 10^{-3}\right.$; $\left.9 \mathrm{~N} 30,6.5 \times 10^{-3} ; 9 \mathrm{~N} 40,5.0 \times 10^{-3} \mathrm{M}\right)$ concentration solutions at $298 \mathrm{~K}$ and transformed to $309 \mathrm{~K}$ assuming that the activation energy of their diffusion is the same as that for water molecules.

The other method-related, systematic error is caused by the obstruction effect of the micelles, which essentially depends on the time $\tau_{\text {obsd }}$ of observation. In NMR, $\tau_{\text {obsd }}=\Delta=5 \times$ $10^{-2} \mathrm{~s}$; the mean distance $r_{\mathrm{D}}$ taken by a water molecule in the bulk phase during $\tau_{\text {obsd }}$ is $r_{\mathrm{D}}=\sqrt{ }\left(D^{0}{ }_{\mathrm{w}} \tau_{\text {obsd }}\right) \sim 1.2 \times 10^{-3} \mathrm{~cm}$. In a micellar solution, the water molecules meet a large number of (impenetrable) micelles within this distance, which cause them to move on average along a longer random path. The observed diffusion coefficient $D^{\prime}$ is $<D^{0}{ }_{\mathrm{w}}$; following ref 33 , they are related to each other via $\Phi$, the volume fraction of the micelles, as

$$
\frac{D^{\prime}}{D_{\mathrm{w}}^{0}} \cong \frac{1}{1+\Phi / 2} \approx 1-\Phi / 2
$$

In a $0.2 \mathrm{M}$ SDS solution, $\Phi=0.048$ and $D^{\prime} / D^{0}{ }_{\mathrm{w}}=0.977$. The systematic error caused by micellar obstruction in the solvent diffusion coefficient is removed in the "net slowing down" $S_{\text {net }}$ defined as

$$
\begin{aligned}
S_{\mathrm{net}}=1-\left(\bar{D}_{\mathrm{w}} / D^{0}{ }_{\mathrm{w}}\right) /\left(D^{\prime} / D^{0}{ }_{\mathrm{w}}\right) \approx \\
1-\bar{D}_{\mathrm{w}} / D^{0}{ }_{\mathrm{w}}-\Phi / 2=S_{\text {obsd }}-\Phi / 2
\end{aligned}
$$

In QENS, $\tau_{\text {obsd }} \sim 10^{-10} \mathrm{~s}$ and $r_{\mathrm{D}} \sim 10^{-7} \mathrm{~cm}$; under these conditions, the obstruction effect can be disregarded and $S_{\text {net }} \approx$ $S_{\text {obsd. }}$ After having calibrated the spectrometer with $D^{0}{ }_{w}$, the 


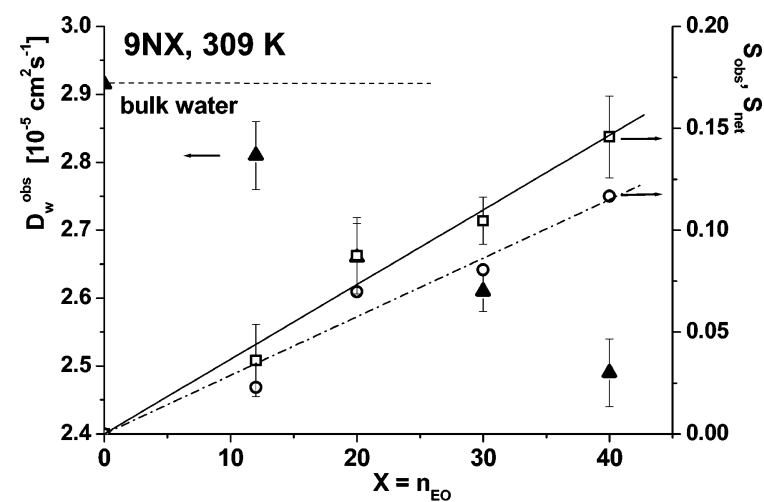

Figure 3. Mean diffusion coefficient $\bar{D}_{\mathrm{w}}(\mathbf{\Lambda})$, observed $S_{\text {obsd }}(\square)$, and net $S_{\text {net }}(O)$ slowing down of solvent molecules obtained by GCSTE NMR in the aqueous phase of $0.04 \mathrm{M} 9 \mathrm{~N} X$ micellar solutions at 309 $\mathrm{K}$ as a function of the number $X$ of ethoxy groups.

diffusion coefficients $D_{\mathrm{m}}$ of the micelles were also determined and listed in Table 2.

\section{Discussion and Conclusions}

3.1. Discussion. The slowing down of water molecules was observed in all micellar solutions we investigated by NMR, and as calculated from the $S_{\text {obsd }}$ and $\Phi$ data in Table $2, S_{\text {net }}>0$ was found in every case. In the particular case of SDS micelles, $S_{\text {net }}$ $=1-0.938 / 0.977=0.040$ from NMR (eq 8), in acceptable agreement with $0.057\left(1-4.33 \times 10^{-6} / 2.98 \times 10^{-5}\right)=0.048$, the QENS result (eq 6). In other words, a net quantity of slow water is present in each micellar system studied and the question arises, why? In ionic systems, the answer would be obvious: due to ionic hydration. This answer, however, is not complete for two reasons. First, in the $0.2 \mathrm{M}$ SDS solution, the concentration of slow $\mathrm{H}_{2} \mathrm{O}$ molecules is $\sim 55.5 \alpha \sim 3.2$ $\mathrm{mol} \cdot \mathrm{dm}^{-3}$ corresponding to $\sim 3.2 / 0.2=16$ slow water molecules per SDS monomer, greater than the sum of the usual hydration numbers of the sodium and sulfate ions. Second, the most significant net slowing down data stem from solutions of nonionic, ethoxylated nonyl phenols (Figure 3); this latter argument is also supported by a former study of ethoxylated surfactant systems ${ }^{14}$ where similar trends were found in the slowing down of solvent molecules versus the length of the ethoxy chains. These findings suggest that, along with ionic hydration, another water-binding mechanism should be considered.

In a molecular dynamics simulation of the cesium pentadecafluorooctanoate micellar system ${ }^{36}$, water molecules are assumed to form $\mathrm{H}$-bonds with the $\mathrm{O}$ atom(s) in the headgroups of the core-forming monomers; this bonding essentially slows down their movement. The network of H-bonds transmits the hindrance in the molecular movement-at a decreasing extenttoward the neighboring solvent molecules. The experimental facts published in the present work seem to support or, at least, do not contradict the existence of this mechanism. According to the simulation, near the micellar surface, the mean squared displacement of the water molecules per unit time (a quantity proportional to their diffusion coefficient) turns out to be definitely less than that in the bulk, ${ }^{36}$ and the ratio of the values in the two regions falls in the range of $D^{\mathrm{s}}{ }_{\mathrm{w}} / D^{0}{ }_{\mathrm{w}}$ obtained from QENS in this work. Atoms, which tend to form H-bonds, are present in the hydrophilic groups of each micellar system: in DTAB, the $\mathrm{N}$ atoms of the trimethylammonium groups, and in SDS and ethoxylated nonyl phenols, the $\mathrm{O}$ atoms of the sulfate groups and the ethoxy chains, respectively. Furthermore, in
Figure $3, \bar{D}_{\mathrm{w}}, S_{\text {obsd }}$, and $S_{\text {net }}$ are proportional to $X$, the number of ethoxy groups in the hydrophilic chains of $9 \mathrm{~N} X$ surfactants.

A further test of QENS results is to compare the thickness $d$ of the hydration layer formed of slow water molecules around the micelles. The quantity $n_{\mathrm{h}}=d / 2 R_{\mathrm{w}}$ is characteristic of the number of hydration layers, where $R_{\mathrm{w}}=3 \sqrt{ }\left(3 V_{\mathrm{w} \Phi} / 4 \pi N_{\mathrm{A}}\right)=$ $0.193 \mathrm{~nm}$ is the mean radius of water molecules calculated from their apparent molar volume $V_{\mathrm{w} \Phi}$. As mentioned before, in a 0.2 M SDS solution, the concentration of slow $\mathrm{H}_{2} \mathrm{O}$ molecules equals $\sim 55.5 \alpha \sim 3.2 \mathrm{~mol} \cdot \mathrm{dm}^{-3}$; calculating with an aggregation number ${ }^{19}$ of 70 , their volume $V_{\mathrm{s}}$ per micelle is $\sim 33.1 \mathrm{~nm}^{3}$. Provided the slow $\mathrm{H}_{2} \mathrm{O}$ molecules form a compact layer around the SDS micellar core of radius ${ }^{19} R_{\mathrm{c}}(=1.57 \mathrm{~nm})$ and axial ratio ${ }^{19}$ $\eta(=1.53), d$ is determined from

$$
V_{\mathrm{s}}=\frac{4 \pi}{3} \eta\left[\left(R_{\mathrm{c}}+d\right)^{3}-R_{\mathrm{c}}^{3}\right]
$$

and equals $0.51 \mathrm{~nm} ; n_{\mathrm{h}}=1.32$ indicates that the number of hydration layers is probably less than 2, in excellent agreement with classical NMR data ${ }^{37}$ obtained in different protein solutions.

Regarding the diffusion coefficient of the micelles, the results from the gradient NMR method follow the expectation: ${ }^{13,15}$ because they have not been corrected for obstruction effects, they are systematically less than (but fall within the same range as) those from QENS and QLS (cf. Table 2).

3.2. Conclusions. QENS experiments made by the neutron backscattering technique support the existence of slowly diffusing solvent molecules in SDS micellar solutions, and conclude both in their diffusion coefficient and mole fraction. Evaluation of the raw data is based on the property of aqueous media that, at the small values of energy and momentum transfer applied, the width of the Lorentzians is well approximated ${ }^{1,2,21}$ by $D Q^{2}$. This condition enables one to fit the model function in the same session to scattering patterns from more than one detector position, thereby improving the information content extracted from the input data. The QENS results on the SDS solution have been confirmed by the GCSTE NMR technique, which was applied to cationic (DTAB) and nonionic (9NX) micellar solutions as well. A net slowing down of water molecules was observed in ionic and nonionic micellar solutions. Consequently, a water-binding mechanism different from ionic hydration should also exist; such a mechanism may be the $\mathrm{H}$-binding of solvent molecules to the $\mathrm{O}$ and $\mathrm{N}$ atoms in the hydrophilic (head)groups of the micelle-forming monomers. ${ }^{36}$ The thickness of the layer formed of slow molecules around the core of SDS micelles is in good agreement with NMR results obtained in protein solutions. ${ }^{37}$

Acknowledgment. Sz.V. and H.G. are indebted to Prof. D. Richter (Institute for Solid State Physics, Jülich Research Center) and Sz.V. and I.B. to Prof. Gy. Batta (University of Debrecen) for valuable discussions. Support from the National Foundation for Scientific Research, Hungary (OTKA), Contract Nos. T038050 (Sz.V.) and T035127 (I.B.), and from the European Access to Research Infrastructure Project (Jülich Neutrons for Europe) is gratefully acknowledged.

\section{References and Notes}

(1) Chen, S.-H.; Teixeira, J.; Nicklow, R. Phys. Rev. A 1982, 26, 3477.

(2) Teixeira, J.; Bellisent-Funel, M.-C.; Chen, S.-H.; Dianoux, A. J. Phys. Rev. A 1985, 31, 1913.

(3) Singwi, K. S.; Sjolander, A. Phys. Rev. 1960, 119, 863.

(4) Chudley, G. T.; Elliot, R. J. Proc. Phys. Soc. 1961, 77, 353.

(5) Sears, P. Can. J. Phys. 1966, 44, 1299; 1966, 45, 237.

(6) Muller, N. Acc. Chem. Res. 1990, 23, 23. 
(7) Hydration Processes in Biological and Macromolecular Systems Faraday Discussions; Royal Society of Chemistry: London, 1996; No. 103.

(8) Nandi, N.; Bagchi, B. J. Phys. Chem. B 1997, 101, 10954.

(9) Denisov, V. P.; Peters, J.; Horlein, H. D.; Halle, B. Nat. Struct. Biol. 1996, 3, 305.

(10) Cabral, J. T.; Luzar, A.; Teixeira, J.; Belissent-Funel, M.-C. Physica B 2000, 276-278, 508.

(11) Calandrini, V.; Deriu, A.; Onori, G.; Lechner, R. E.; Pieper, J. Appl. Phys. A 2002, 74 (Suppl.), S1339.

(12) Almássy, L.; Bánki, P.; Bellisent-Funel, M.-C.; Bokor, M.; Cser, L.; Jancsó G.; Tompa, K.; Zanotti, J. M. Appl. Phys. A 2002, 74 (Suppl.), S1376.

(13) Nilsson P. G.; Lindman, B. J. Phys. Chem. 1983, 87, 4756.

(14) Fukuda, K.; Söderman, O.; Lindman, B.; Shinoda, K. Langmuir 1993, 9, 2921.

(15) Popova, M. V.; Tchernyshev, Y. S.; Michel, D. Langmuir 2004, 20,632 .

(16) Van Hove, L. Phys. Rev. 1954, 95, 249

(17) Egelstaff, A. An Introduction to the Liquid State; Academic Press: London, 1967.

(18) Vineyard, G. H. Phys. Rev. 1958, 110, 999.

(19) Vass, Sz.; Borbély, S.; Gilányi, T. J. Phys. Chem. B 2000, 104, 2073.

(20) Sears, V. F. Thermal-Neutron Scattering Lengths and Cross Sections for Condensed Matter, Report AECL-8940; Chalk River Nuclear Laboratories: Chalk River, Ontario, 1984.

(21) Water. A Comprehensive Treatise; Franks, F., Ed.; Plenum Press: New York, 1972; Vol. 1.

(22) Weingärtner, H. Z. Phys. Chem. 1982, 132, 129.
(23) Hayter, J. B.; Penfold, J. SQHP, a FORTRAN Package to Calculate $S(Q)$ for Macroion Solutions, ILL Report 80HA07S; Grenoble, 1980. Hayter, J. B.; Penfold, J. The Structure Factor of Charged Colloidal Dispersions at any Density, ILL Report 82HA14T; Grenoble, 1982.

(24) Hayter, J. B.; Penfold, J. J. Chem. Soc., Faraday Trans. 1 1981, 77,1851 .

(25) Corti, M.; Degiorgio, V. J. Phys. (Paris) 1981, 85, 711.

(26) Arrighi, V.; Holmes, P. F.; Cagliardi, S.; McEwen, I. J.; Telling, M. T. F. Appl. Phys. A 2002, 74 (Suppl.), S466.

(27) Pelta, M. D.; Barjat, H.; Morris, G. A.; Davis, A. L.; Hammond, S. J. Magn. Reson. Chem. 1998, 36, 706.

(28) Stilbs, P. Prog. NMR Spectrosc. 1987, 19, 1.

(29) Johnson, C. S., Jr. Prog. NMR Spectrosc. 1999, 34, 203.

(30) Stejskal, E. O.; Tanner, J. R. J. Chem. Phys. 1965, 42, 288.

(31) Söderman, O.; Stilbs, P. Prog. NMR Spectrosc. 1994, 26, 445 Söderman, O.; Stilbs, P.; Price, W. S. Concepts Magn. Reson. A 2004, 23, 121.

(32) Furó, I. J. Mol. Liq. 2005, 117, 117.

(33) Bell, G. M. Trans. Faraday Soc. 1965, 60, 1752. Jönnson, B.; Wennerström, H.; Nilsson, P. G.; Linse, P. Colloid Polym. Sci. 1986, 264 77.

(34) Vass, Sz.; Török, T.; Jákli, Gy.; Berecz, E. J. Phys. Chem. 1989, 93,6553 .

(35) Perron, G.; Desrosiers, N.; Desnoyers, J. E. Can. J. Chem. 1976 $54,2163$.

(36) Balasubramanian, S.; Bagchi, R. J. Phys. Chem. B 2002, 106, 3668 .

(37) Halle, B.; Andersson, T.; Forsén, S.; Lindman, B. J. Am. Chem. Soc. 1981, 103, 500. 